\title{
Research in Interactive Drama Environments, Role-Play and Story-Telling
}

\author{
Ruth Aylett, Sandy Louchart, and Allan Weallans \\ MACS, Heriot-Watt University, Riccarton, Edinburgh EH14 4AS \\ \{r.s.aylett, s.louchart, aw119\}@hw.ac.uk
}

\begin{abstract}
This paper gives an overview of the UK network RIDERS Research in Interactive Drama Environments, Role-Play and Story-telling, running for 36 months from September 2011. It discusses the three central themes of RIDERS: theoretical work on the conflict between interactivity and narrative content, problems, issues and tools relating to authoring, and directions in evaluation. It gives a brief overview of the current position in each of these areas and suggests how RIDERS activity might be able to contribute to them. Finally it summarises the overall RIDERS programme of activity.
\end{abstract}

Keywords: Interactive storytelling, Narrative engagement, Authoring, Evaluation.

\section{Introduction}

RIDERS (Research in Interactive Drama Environments, Role-Play and Story-telling) is a network funded by the UK Research Councils for three years from September 2011 to bring together researchers in interactive drama environments, role-play and story-telling in a series of events. The case for RIDERS rests on the fact that these areas are researched and applied in a wide range of disciplines.

Within AI, storytelling research began with NLP work on story-grammars in the 1970s [9], aimed at the non-interactive generation of text-based stories. For example, Meehan's TALESPIN [34] used character goals and planning to produce very short fable-like stories.

However there is of course a substantial background relating to interactive storytelling and narrative in many other disciplines, often over a much longer period. Narratology - theoretical discussion on the nature of story - goes back in the west to Aristotle; and there is also extensive work in psychology (especially around autobiographic memory); drama (Improv and interactive theatre); film and television; education and training (role-play; experiential learning) and art and digital-media (interactive installations). Within computer science too, there is more generic work on interaction and its impact on the user in the field of HCI. This reflects the central importance of story in human culture and society as well as in the sense of self of the individual. 


\section{And the Holodeck?}

The advent of multi-media systems and then Virtual Reality - immersive real-time interactive graphic environments - in the 1990s, created a new vision of a dramatic 'holodeck'-like experience [7,35] or virtual theatre [20]. Here a highly-immersed user could act as a character interacting with other artificial characters in an interactive graphical narrative experience qualitatively different from existing media - whether novels, theatre or film. With the advent of mobile technology and augmented reality, this vision has further extended into interactive dramatic or narrative experiences in which the real world would also be a component $[8,24]$.

Realisation of this vision of interactive storytelling (IS) would be a highly significant research achievement with substantial real-world impact - story-based education and training systems, completely new entertainment genres, serious games, novel therapy systems, creative new artistic and performance works. The computer game industry in particular has become very conscious of the need for narrative engagement as a path to a wider gamut of player emotions than those generated by destroying virtual enemies. However, in spite of a small number of working prototype systems, primarily in the US [e.g. 3, 30, 42, 46], and a smaller number in Europe [e.g. 4, 13, 37], the vision is still substantially unrealised. Here we summarise some possible reasons, and discuss each in a little more detail in succeeding sections.

Reasons for relatively slow progress are both theoretical and practical. One fundamental challenge is how to resolve the clash between the interactive freedom expected by the user in such environments and an authorial demand for guaranteed narrative structure. Computer games have often avoided the problem altogether by using non-interactive 'cut' scenes for narrative content, effectively isolating the substance of the narrative from the interaction of the gameplay. Less commonly, preauthored branching structures have been applied, allowing the user a limited degree of interactive freedom by offering a controlled set of choices the author can anticipate. The promise of AI-based generative techniques has been so far largely ignored, with very few exceptions (The Sims, Spore).

The big advantage of a generative approach is that it offers compact representations that can produce a very large story world. Thus an AI planning system can instantiate the variables in the action repertoire it is using with any of the set of acceptable instances in a world, where this set could potentially be very large. One abstract action can then represent a much bigger set of instantiated concrete actions. However, abstract AI representations have no more communicative power for spectator/participants in an interactive narrative than they do for creative artists. An AI planning template expressed in logical form is equally impenetrable to both. Thus generative approaches compound the challenge of how to support authors in applying such novel technologies when they necessarily have no interest in understanding how an AI planner works or the technical parameters determining character personality and affect $[22,27,30]$.

They also raise the question of how, once instantiated, generative AI outputs can be compellingly presented, whether in language or in animation. This is one cause of the significant gulf between researchers and both creative artists and potential 
industrial applications that inhibits the take-up of successful research innovations in the field. A closely related challenge is whether and how standardised components and formalisms can be created allowing the sharing of research, the building of new systems by extending older ones and the reuse and adaptation of content.

A third significant challenge lies in evaluation - how to measure the success of an IS system and to compare one system to another. The traditional HCI measure of usability is defined as the ease with which a user can employ a particular computerbased tool to achieve a particular goal. While poor usability could certainly break the immersion of a user in a narrative experience, making it a necessary condition for a good IS system, it does not help much in evaluating the quality of the stories (or 'experiences') that are produced, making it plainly insufficient. Recent work in HCI on 'the user experience' [23] is more promising in this respect. A simple measure of length of narrative experience [13] has been suggested, as have metrics from the Humanities [40] and work on user engagement in computer games [16, 49].

Solutions to these and other challenges are inherently inter-disciplinary. Thus AI and graphics researchers have drawn on various narrative and dramatic theories from the humanities - Aristotle [29], Boal [4, 10] and Propp [37, 38] being only three of many. However, often when one field borrows from another, it grabs ideas at a partial/superficial level based on its own limited understanding. IS research needs to dig deeper in understanding related arts and humanities research domains as well as the psychology of the user.

At the same time, IS has a lot to offer such related research fields, both in concretising and refining theoretical concepts in psychology, arts and humanities, and in developing technologies that support new creative possibilities for practitioners. Thus all would benefit from interacting, through systematic debate and collaborative design activities that are currently only taking place through personal contacts and chance meetings. RIDERS will take a community building and systematic approach in exploring common and relevant areas and develop a common understanding of issues and solutions through mediation and interaction.

\section{Structure and Interaction}

That the characteristic feature - interactivity - of this field should contradict a basic feature of narrative, namely pre-authored causal structure, is a narrative paradox within which nearly all IS research struggles. It can be recast as a conflict between plot and an autonomous character given that a user actively participating in an interactive narrative can be thought of as a character whose actions need not be those selected by a prior plot.

Forster [17] argued that 'the king died and then the queen died' is merely a chronological sequence of events, while 'the king died and then the queen died of grief' is a plot because it includes a causal link between the events. Less often commented upon in this example is that the suggested cause is an affective change in one of the characters. Many narrative formalisms have omitted character affective state altogether because of their focus on world-based causal structure [29, 38], and while the concept of an internal reaction in story grammar work [2] partially covers 
this idea, it is largely used to capture reactions rather than as part of a character action-generation system with full causal weight.

One possible view of the unfolding process of a story is that of an iteration between events in the world and affective changes in the characters that are both responses to events and causes of them. Causal chains that contain no affective impact upon characters are arguably more like the problem-solving of adventure games than narrative, while affective change in characters with no causal impact on the world are more like social web environments than narrative.

This idea can provide a framework for thinking about the different attempts to solve the paradox.'

Thus some researchers have investigated how pre-authored plot can be dynamically modified in response to user actions [39], intervening at the point of the CAUSE between Character Actions and World State. Character autonomy is unaffected but the outcomes of character actions may not be those they intended. An alternative approach starts from the CAUSE between events and affective states and focuses on action selection mechanisms for intelligent expressive characters. In this case emergent structure could be created $[4,12]$.

The two approaches have inverse advantages and disadvantages, as is true in general in AI when one considers top-down and bottom-up approaches. The top-down approach begins with a plot (whether pre-written or machine generated), and it is this that directs the characters' actions. The main advantage of this approach is that it maintains a coherent narrative structure and embeds dramatic interest into this structure. However the disadvantage is that the plot may direct the characters to perform actions that are not believable, especially where replanning takes place to accommodate unexpected user actions.

This may be in relation to the character's nature or capabilities: for example, if the plot turns out to require that a quiet, shy character jumps onto a table

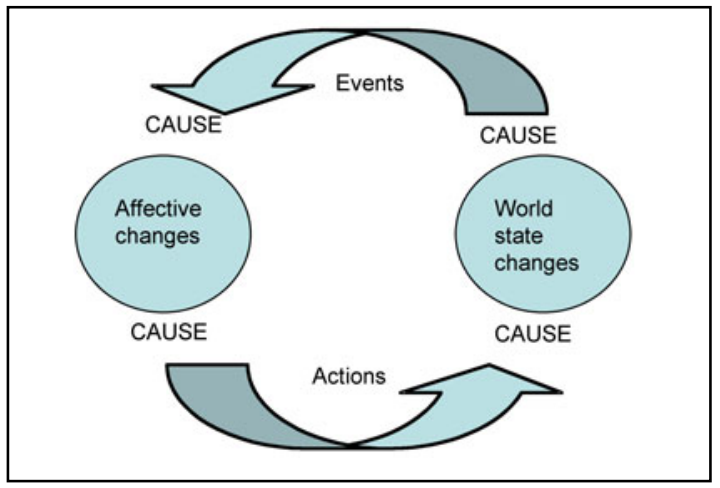

Fig. 1. A narrative loop and dances, that a crack shot misses their target or a heavily overweight character sprints a kilometre. Of course a character transgressing their basic nature may be a fertile source of narrative surprise and interest, but this requires that sufficient motivation to do so has been established as part of the character's arc, or that the violation is causally justified retrospectively. This can be difficult: if the crack shot must miss because their target is essential to the plot later, and if their response is to shoot again, the pre-determined nature of the plot becomes all too visible. 
Similar problems arise in relation to creating inconsistencies in the character's current context. For example, in a scene with two terrorist characters, but where a known CIA agent character is in fact present, the plot may require the terrorists to have some dialogue discussing an upcoming attack, even though believability suggests that they would not want the CIA agent to overhear. A top-down approach may also simply interdict player actions that violate the plot, limiting the player's agency in a way that can be obvious enough to destroy their narrative engagement.

The bottom-up approach starts with autonomous character action-selection with an emphasis on acting believably according to their beliefs, desires and intentions, their emotional state, and their situational context. However in a pure in-role simulation approach, characters will favour the most realistic actions, and these may not be dramatically interesting [26, 45]. Even if a character does perform one dramatically interesting action, it is less likely that it will perform a sequence of dramatically interesting actions constituting a dramatically recognisable story, and less likely still that all characters will do so.

This is because characters have no dramatic intelligence: they do not "know" they are trying to tell a story; all they "know" is that they are trying to simulate a human role. In essence, such an agent's action-selection mechanism works entirely on the incharacter (IC) level, while the notion of drama is an out-of-character (OOC) concern: the characters themselves are not concerned about the drama and do not take on dramatic responsibilities. They participate in the cycle of Figure 1 but do not model it.

One can contrast here the role of actors in improvisational drama, where the concept of an 'offer' - actions giving scope for mutual dramatic development - is a basic one [41]. Current approaches to incorporating dramatic intelligence into characters' action selection are based on generating a range of possible actions rather than just one at each time step and using a metric for selecting between them that involves dramatic responsibility. An initial step might be to choose the most dramatic possible action from the available set, for example by reapplying cognitive appraisal ('double appraisal') to determine its potential affective impact [25]. However this neglects the requirement for pacing in the dramatic trajectory of an interactive drama and more recent work seeks to distribute dramatic responsibility into a multi-agent architecture [48].

Both plot- and character-based approaches have looked at the role of story management, facilitation or direction [6, 27, 30,47], which can be placed at any of the four CAUSE points in Figure 1. RIDERS has a part to play in supporting a growing investigation of story-shaping in genres such as improvisational drama [41, $45]$ and table-top role-play [6, 47].

\section{Authoring Challenges}

No matter how ingenious solutions to the reconciliation of structure and interaction might be, to turn them into interactive narratives requires authoring of content. The first issue here is that exactly what should be authored depends on the architecture of the run-time system. Thus for example, Comme Il Faut [31] requires the writing of a large set of social rules; FearNot! [4] requires the setting of character emotional 
parameters and the definition of AI planning action repertoires. To do this successfully requires an understanding of how these formalisms will be translated into actual story by the run-time system, and thus a background in AI technology that is never going to be widely available.

A second issue is that moving outside of linear stories with a single causal chain of events requires an increase in the amount of content while at the same time distancing the authoring of such content from the context of its presentation by the run-time system. This is not an approach to authoring generally applied in media, with the exception of live and table-top role-play. In the latter, campaigns are frequently designed within rich declaratively-described story-worlds represented in one or more handbooks that can be reused. These handbooks serve to define not a story in itself, but the potentials and boundaries of stories within that scenario. This can be likened to what Bruckman [11] has called a 'storyspace': "a combination of temporal, spatial and thematic mappings" that stores information about potential stories. However, the lack of representational standards for combined AI and graphical systems make the development of a database of equivalent story-world material a currently infeasible task.

A third issue is that the production of content for an interactive graphical narrative is unlikely to be a single-author task: the skill of story-writing and the skill of animation are quite different. In both film (linear narrative) and computer games (branching narrative at best) screen-play/branch flow are authored by separate team members from those making actual film footage or creating animations. Research teams usually lack the necessary range of expertise and resources, while commercial teams are disinclined to take the risks of unproven approaches.

If we view an interactive narrative as a type of knowledge-based system (KBS),then it should come as no surprise that authoring is a difficult task, since it corresponds to knowledge acquisition, long the bottleneck in the construction of KBSs. In the KBS world, the initial approach taken was that of a knowledge engineer, familiar with the AI formalisms, working with an expert, responsible for the content. This handcrafting approach has always proved problematic except for very small systems, and is therefore not a good model for interactive narrative either.

Two other broad approaches have been tried in the KBS world. One is to embed a strong model into an authoring tool that is used by the expert themselves. The key idea here is that the model allows the expert to conceptualise and structure their content at a problem-based level rather than at an AI technology level. If the model is appropriate to the expert's needs this approach can work well, and it was widely applied in medical diagnosis applications. In a weak form it may be found in the authoring tools supplied with games such as NeverWinter Nights, where as long as the user wants a story based on encounters with monsters with a few branching choices an application can be produced quickly using the database of graphical materials supplied. A number of researchers with run-time IS systems have produced authoring tools for them [e.g. 15, 21, 31, 33 and others] but without much evidence of take-up. In the absence of reported usability studies a plausible assumption is that these do not operate at a sufficiently abstracted level to allow authoring without the detailed understanding of the run-time system's technology already discussed. 
The other approach developed in the KBS world rests on machine learning technologies. Here the basic idea is that structured knowledge can be induced from examples, whether given directly by the expert, extracted from existing data records, or by real-time monitoring of an expert in action. A lesson from the KBS work is that learning rules is much easier than learning more structured representations such as AI planning operators. Thus Thespian, using decision theoretic representations, is able to learn character representations [43], but there are few other examples. Induction does of course work better with greater amounts of data than a single user may be motivated to provide, and some recent approaches use web access to gather data from multiple users $[22,36]$.

What can RIDERS contribute to this difficult area? Again, inter-disciplinary studies seem useful; support for standardisation activities that would allow reuse of materials across different run-time systems would also be sensible. Some fields have produced greater cohesion by running competitions involving a standard benchmark problem or game engine, and the use of a common story - Little Red Cap - in authoring workshop [44] suggests this should also be pursued. Public availability of data on which narrative learning algorithms could be tried out may also be a worthwhile activity.

\section{Evaluation}

Evaluation is a seriously under-developed area in IS work, but again the difficulties involved offer some reasons for this. If technology is immature or content is scanty, evaluation risks only assessing these weaknesses. Research systems may derive their standing within the community from video clips shown during paper presentations, while the experience of an engaged user may be quite different [5]. While in general one might like to evaluate the user experience [23] intuitively, metrics relating to a specifically narrative experience appear desirable.

To evaluate the impact of an interactive narrative, one must consider what 'impact' means for this form, and whether it is the same as 'impact' in traditional, noninteractive stories, as studied in literary theory. The tendency of new media to think of themselves within the constraints of older forms is well-described in [32]:

"New media are new archetypes, at first disguised as degradations of older media. These degradations happen when new media inevitably use old media as content. Using the older ones as content hastens the tidying up process by which a medium becomes an art form."

This phenomenon is summed up in McLuhan's famous phrase, "the medium is the message," and can be seen in past media that have since matured. Early cinema was little different to a recorded stage play, while early television drama took the form of a visual radio play. Cinema and television now make use of the strengths of their own media, rather than trying to model other forms. The same phenomenon undoubtedly affects interactive digital media: many commercial video games take cinematic films as their model, while attempting to create an "interactive movie" has occurred more than once in the history of video games, with limited success [1]). For the medium to mature, the properties that make it unique from other media must be investigated. 
In traditional narrative, the Aristotelian impact, or tension, is focused on the plot of the story. However, the difference between interactive narrative and non-interactive narrative is of course interactivity: the involvement of the user. One must question whether focusing the evaluation of impact on the plot is appropriate in an interactive medium, or whether it is using "old media as content". Here it seems much more appropriate to focus on evaluating the impact on the user. This is the way Game Masters in table-top role playing games behave: their concern is more for whether the impact of the events in the story is satisfying for their players, and less for whether the events of the game would read as a satisfying story after the fact, were someone to write them down [47].

Like any other subjective experience, narrative experience evaluation faces the issue of post-hoc rationalization if post-experience questionnaires are used, along with under-developed or ambiguous objective measures, whether observational or via the capture of physiological response data. It may be necessary to infer engagement rather than directly measure it [16]. Embedding evaluation into the user's narrative interaction [19] is one approach, learning patterns of user behaviour and trying to match these to post-hoc user evaluation data [49] is another that has been tried in computer game research.

This type of evaluation attempts a holistic assessment of a specific interactive narrative. It cannot be used on the one hand to compare different interactive narratives or on the other to establish whether specific technological innovations do or do not contribute to its degree of success.

Comparison between IS systems may in fact be utopian, or at least as subjective as the one star- five star assessments given by newspapers to films. Assessment of technology features is more feasible. For example, if an aim of an IS system is to produce empathy with its characters, as was the case in FearNot![4], then one can draw on methods from psychology to evaluate how far this is so [18]. In the same way, if an IS system is using replanning to move the narrative back towards an intended plot, one can evaluate the user's reaction to those events in the story and their impact on believability.

The development of conceptual models for IS systems, at a more abstract level than the implementational formalisms, might also allow some more generic approaches to classes of evaluation. Thus in Figure 1 above, just as one could apply story management techniques to each of the four CAUSE links (world state-events; events-character-affective state; character affective state-actions; actions-world state), so one could apply evaluation metrics of stability, believability, dramatic impact. Defining conceptual dramatic trajectories (temporal patterns absent from Figure 1) would also have an impact on generic evaluation approaches.

Events such as ICIDS already allow both evaluation techniques and the outcomes of evaluation to be shared among IS researchers. Where a network like RIDERS may be able to help is in increasing access to methods from other disciplines, whether those of ethnography and psychology, or literature and film.

\section{RIDERS Activities}

Unlike IRIS [14], which is funded by the EU, RIDERS has a specifically UK remit. Nevertheless it has argued successfully that developing IS in the UK also involves 
strengthening links with international research. RIDERS will seek to work not only with IRIS but also with the European Narratology Network (http://www.narratology.net/) and the Serious Games network GALA (http://www.galanoe.eu/ ), as well as IS research groups throughout the world. To this end it includes funding of four one week visits by UK researchers to international centres of excellence, for which there will be open calls as soon as the RIDERS Steering Committee has been set up.

Where IRIS includes funding for research work, RIDERS, like all UK-funded networks, is only funded for the organisation of events. Thus it cannot itself offer direct solutions to the research challenges discussed above, and will only adopt a specific stance itself insofar as its members arrive at consensus: friendly debate and honest differences will help to develop the IS field.

RIDERS will support three main types of activity: 1) A six meeting programme of events for network members on the three themes identified above; 2) Exchange visits to both UK and international labs which will enable researchers to discuss the different contexts and disciplines of interactive drama, role play and storytelling and visit relevant innovative labs/studios in academia or industry; 3) Support for PhD students to visit conferences to present work on topics related to RIDERS interests. Finally, its program includes the organisation of an IS summer school in 2013 or 2014.

RIDERS will also develop a website which it hopes will become a useful resource for the whole research community; it is committed to a set of legacy materials including a book.

Events and community-building do not themselves solve research problems, but if they result in new collaborations and the generation of new ideas, they may help to do so. On Structure and Interaction, exposing IS researchers to non computer-based forms of interactive narrative and drama can provide new inspirations. The Improv sessions in recent AAAI Fall Symposia Interactive Narrative workshops have had the effect of both increasing understanding of this form and giving the work of researchers involved in taking this into computational modelling a higher profile. One of the RIDERS meetings is projected to take the form of a live role-play weekend with the aim of providing a similar understanding for IS researchers of this interactive form. The initial members of RIDERS already span arts and humanities researchers, and opportunities to draw on the experience of practitioners will also be facilitated.

Multi-disciplinary teams have been identified above as one of the requirements for attacking issues relating to authoring. RIDERS will attempt to act as a broker between practitioners wanting to construct interactive narratives and members wanting to apply their technology to a specific domain. Again, its two meetings relating to this theme will not take the standard academic form of paper presentation but will aim to produce authoring experiences, bringing members together with one or more standard story worlds for which some material content can be supplied. The website will be developed to include a repository for such materials to allow those entering the field to make a much quicker start than is currently possible. The evaluation theme will be similarly supported with meetings in which multiple evaluation approaches will be tested out and the website will include as comprehensive a listing as possible of available techniques from all of the component disciplines of its members. 


\section{References}

1. Adams, E.: Interactive Narratives Revisited: Ten Years of Research - (2005), GDC talk: http://www.designersnotebook.com/Lectures /

Interactive_Narratives_Revisit/

interactive_narratives_revisit.htm (last visited July 07, 2011)

2. Anderson, K., Evans, C.: The development of the canonical story grammar model and its use in the analysis of beginning reading computer stories. Reading Improvement 33, 2-15 (1996)

3. Anstey, J.: The Thing Growing (2000), http: / / bcchang.com/immersive_blog/ ?p=524 (accessed June 27, 2011)

4. Aylett, R.S., Vala, M., Sequeira, P., Paiva, A.: FearNot! - an emergent narrative approach to virtual dramas for anti-bullying education. In: Cavazza, M., Donikian, S. (eds.) ICVSVirtStory 2007. LNCS, vol. 4871, pp. 202-205. Springer, Heidelberg (2007)

5. Aylett, R.S., Louchart, S.: Being There: Participants and Spectators in Interactive Narrative. In: Cavazza, M., Donikian, S. (eds.) ICVS-VirtStory 2007. LNCS, vol. 4871, pp. 117-128. Springer, Heidelberg (2007)

6. Aylett, R.S., Louchart, S., Tychsen, A., Hitchens, M., Figueiredo, R., Delgado Mata, C.: Managing Emergent Character-Based Narrative. In: Proceedings of INTETAIN 2008 (2008)

7. Bates, J.: Virtual reality, art, and entertainment. Presence: The Journal of Teleoperators and Virtual Environments 1(1), 133-138 (1992)

8. Benford, S., Crabtree, A., Reeves, R., Flintham, M., Drozd, A., Sheridan, J.G., Dix, A.: The frame of the game: blurring the boundary between fiction and reality in mobile experiences. In: CHI 2006, pp. 427-436. ACM (2006)

9. Black, J.B., Wilensky, R.: An evaluation of story grammars. Cognitive Science 3, 213-230 (1979)

10. Boal, A.: The Theatre of the Oppressed. Urizen Books, New York (1979)

11. Bruckman, A.: The Combinatorics of Story-Telling: Mystery Train Interactive. Interactive Cinema Group internal paper, MIT Media Lab (1990),

http://mf.media.mit.edu/pubs/techreport/Combinatorics.pdf (accessed September 8, 2011)

12. Cavazza, M., Charles, F., Mead, S.J.: Character-based Interactive Storytelling. IEEE Intelligent Systems, Special Issue on AI in Interactive Entertainment, 17-24 (2002)

13. Cavazza, M., Lugrin, J.-L., Pizzi, D., Charles, F.: Madame Bovary on the holodeck: immersive interactive storytelling. ACM Multimedia, 651-660 (2007)

14. Cavazza, M., Champagnat, R., Leonardi, R.: The IRIS Network of Excellence: Future Directions in Interactive Storytelling. In: Iurgel, I.A., Zagalo, N., Petta, P. (eds.) ICIDS 2009. LNCS, vol. 5915, pp. 8-13. Springer, Heidelberg (2009)

15. Crawford, C.: Storytron Interactive Storytelling, Project Website: http: / /www. storytron. com (accessed July 6, 2011)

16. Fischer, J.E., Benford, S.: Inferring player engagement in a pervasive experience. In: Proc. of CHI 2009. ACM Press (2009)

17. Forster, E.M.: Aspects of the Novel (1927)

18. Hall, L., Jones, S., Hall, M.: Investigating user engagement with synthetic characters. In: Personal, Social and Emotional Virtual Learning Environments, ICALT 2008, Santander, Spain, July 1-5 (2008) 
19. Hall, L., Aylett, R.S., Paiva, A.: An Immersive Approach to Evaluating Role Play. In: Ruttkay, Z., Kipp, M., Nijholt, A., Vilhjálmsson, H.H. (eds.) IVA 2009. LNCS, vol. 5773, pp. 498-499. Springer, Heidelberg (2009)

20. Hayes-Roth, B.R.: Acting in Character. In: Trappl, R., Petta, P. (eds.) Creating Personalities for Synthetic Actors. LNCS, vol. 1195. Springer, Heidelberg (1997)

21. Iurgel, I.: Cyranus - An Authoring Tool for Interactive Edutainment Applications. In: Pan, Z., Aylett, R.S., Diener, H., Jin, X., Göbel, S., Li, L. (eds.) Edutainment 2006. LNCS, vol. 3942, pp. 577-580. Springer, Heidelberg (2006)

22. Kriegel, M., Aylett, R.S.: A mixed initiative authoring environment for emergent narrative domains. In: Proc., AISB Symposia 2007, Univ. Newcastle, pp. 453-456 (April 2007)

23. Law, E., Roto, V., Hassenzahl, M., Vermeeren, A., Kort, J.: Understanding, Scoping and Defining User Experience: A Survey Approach. In: Proceedings of Human Factors in Computing Systems Conference, CHI 2009, Boston, MA, USA, April 4-9 (2009)

24. Lim, M.Y., Aylett, R.S., Enz, S., Kriegel, M., Vannini, N., Hall, L., Jones, S.: Towards Intelligent Computer Assisted Educational Role-Play. In: Chang, M., Kuo, R., Kinshuk, Chen, G.-D., Hirose, M. (eds.) Learning by Playing. LNCS, vol. 5670, pp. 208-219. Springer, Heidelberg (2009)

25. Louchart, S., Aylett, R.S., Dias, J.: Double Appraisal for Synthetic Characters. In: Pelachaud, C., Martin, J.-C., André, E., Chollet, G., Karpouzis, K., Pelé, D. (eds.) IVA 2007. LNCS (LNAI), vol. 4722, pp. 393-394. Springer, Heidelberg (2007)

26. Louchart, S., Swartjes, I., Kriegel, M., Aylett, R.S.: Purposeful Authoring for Emergent Narrative. In: Spierling, U., Szilas, N. (eds.) ICIDS 2008. LNCS, vol. 5334, pp. 273-284. Springer, Heidelberg (2008)

27. Loyall, B., Reilly, S., Bates, J., Weyhrauch, P.: System for authoring highly interactive, personality-rich interactive characters. In: Proceedings of the 2004 ACM SIGGRAPH / Eurographics Symposium on Computer Animation (2004)

28. Magerko, B., Laird, J.E., Assanie, M., Kerfoot, A., Stokes, D.: AI Characters and Directors for Interactive Computer Games. In: Procedings of the 16th National Innovative Applications of AI Conference (2004)

29. Mateas, M.: A preliminary poetics for interactive drama and games. In: Proceedings of SIGGRAPH 2001, Art Gallery, Art and Culture Papers, pp. 51-58. ACM Press, New York (2001)

30. Mateas, M., Stern, A.: Structuring content in the Façade interactive drama architecture. In: Proceedings of Artificial Intelligence and Interactive Digital Entertainment, Marina del Rey (2005)

31. McCoy, J., Treanor, M., Samuel, B., Tearse, B., Mateas, M., Wardrip-Fruin, N.: Authoring Game-Based Interactive Narrative using Social Games and Comme il Faut. In: Proc. 4th Int. Conf. \& Festival of the Electronic Literature Organization: Archive \& Innovate (2010)

32. McLuhan, M.: Understanding Media: The Extensions of Man, 1st edn. McGraw Hill, NY (1964); reissued MIT Press (1994)

33. Medler, B., Magerko, B.: Scribe: A Tool for Authoring Event Driven Interactive Drama. In: Göbel, S., Malkewitz, R., Iurgel, I. (eds.) TIDSE 2006. LNCS, vol. 4326, pp. 139-150. Springer, Heidelberg (2006)

34. Meehan, J.R.: The Metanovel: Writing Stories by Computer. PhD dissertation, Yale University (1976)

35. Murray, J.: Hamlet on the Holodeck. MIT Press, Cambridge (1998)

36. Orkin, J., Smith, T., Reckman, H., Roy, D.: Semi-Automatic Task Recognition for Interactive Narratives with EAT \& RUN. In: Proceedings of the 3rd Intelligent Narrative Technologies Workshop, 5th Int. Conf. on Foundations of Digital Games, FDG (2010) 
37. Prada, R., Machado, I., Paiva, A.: TEATRIX: Virtual Environment for Story Creation. In: Gauthier, G., VanLehn, K., Frasson, C. (eds.) ITS 2000. LNCS, vol. 1839, pp. 464-473. Springer, Heidelberg (2000)

38. Propp, V.: Morphology of the Folktale. University of Texas Press (1968)

39. Riedl, M., Michael Young, R.: An intent-driven planner for multi-agent story generation. In: 3rd Int. Conf. on Autonomous Agents and Multi Agent Systems (July 2004)

40. Riedl, M., Young, R.M.: An Objective Character Believability Evaluation Procedure for Multi-agent Story Generation Systems. In: Panayiotopoulos, T., Gratch, J., Aylett, R.S., Ballin, D., Olivier, P., Rist, T. (eds.) IVA 2005. LNCS (LNAI), vol. 3661, pp. 278-291. Springer, Heidelberg (2005)

41. Riedl, M.: A Comparison of Interactive Narrative System Approaches Using Human Improvisational Actors. In: Proceedings of the Third Workshop on Intelligent Narrative Technologies, Monterey, California (2010)

42. Rowe, J., Mott, B., McQuiggan, S., Robinson, J., Lee, S., Lester, J.: Crystal Island: A Narrative-Centered Learning Environment for Eighth Grade Microbiology. In: Workshop on Intelligent Educational Games at the 14th International Conference on Artificial Intelligence in Education, Brighton, U.K, pp. 11-20 (2009)

43. Si, M., Marsella, S., Pynadath, D.V.: Thespian Using multi-agent fitting to craft interactive drama. In: Proc. AAMAS 2005 (2005)

44. Spierling, U., Iurgel, I.: Pre-Conference Demo Workshop "Little Red Cap": The Authoring Process in Interactive Storytelling. In: Göbel, S., Malkewitz, R., Iurgel, I. (eds.) TIDSE 2006. LNCS, vol. 4326, pp. 193-194. Springer, Heidelberg (2006)

45. Swartjes, I., Kruizinga, E., Theune, M.: Let's Pretend I Had a Sword: Late Commitment in Emergent Narrative. In: Spierling, U., Szilas, N. (eds.) ICIDS 2008. LNCS, vol. 5334, pp. 264-267. Springer, Heidelberg (2008)

46. Swartout, W., Hill, R., Gratch, J., Johnson, L., Kyriakakis, C., Labore, C., Lindheim, R., Marsella, S., Miraglia, D., Moore, B., Morie, J., Rickel, J., Thiebaux, M., Tuch, L., Whitney, R., Douglas, J.: Towards the holodeck: integrating graphics, sound, character and story. In: Proc. 5th Int. Conf. on Autonomous Agents (2001)

47. Tychsen, A., Hitchens, M., Aylett, R.S., Louchart, S.: Modeling Game Master-based story facilitation in multi-player Role- Playing Games. In: AAAI Spring Symposium Interactive Narrative Technologies II Stanford USA (2009)

48. Weallans, A., Louchart, S.: Distributed drama management: integrating levels of narrative responsibility. In: Proceedings, Artificial Intelligence and Simulated Behaviour Symposia AISB 2010 Leicester UK (2010)

49. Yannakakis, G.N.: Learning from Preferences and Selected Multimodal Features of Players. In: Proceedings of the Int. Conf. on Multimodal Interfaces (ICMI 2009). ACM Press, Cambridge (2009) 